The diverse patient and dialysis-unit characteristics in the United States pose challenges for assessing the safety and efficacy of reuse practices. Collins and coinvestigators from the Hennepin County Medical Center, University of Minnesota, conducted a study to determine if the chemical germicides used to sterilize the dialyzers during reprocessing had an effect on patient mortality.

A $10 \%$ random sample of period-prevalent hemodialysis patients from units practicing conventional dialysis ( $<25 \%$ of patients with high-efficiency/high-flux dialysis) were analyzed. The data included 13,926 patient observations in 1989-1990 and 20,422 in 1991-1993. CDC-Health Care Financing Administration facility survey Medicare data were analyzed with a Cox regression model, evaluating the risk of reuse compared with no reuse and adjusting for comorbidity, unit characteristics, and profit status. In 1989-1990, freestanding and hospital-based units that did not reuse dialyzers were not significantly different from each other in mortality rates. In 1991-1993, however, noreuse, freestanding, for-profit units had higher risks (relative risk $[R R]=1.23, P=.003$ ) compared with no-reuse, hospital-based, nonprofit units. No-reuse, hospital-based, forprofit units, in contrast, were associated with a lower mortality risk ( $\mathrm{RR}=0.70, P=.0001)$.

An isolated higher risk associated with peracetic acid manual reuse in freestanding units (1989-1990) was identified in for-profit units only. In the 1991-1993 period, an increased mortality risk was noted in hospital-based nonprofit units practicing formaldehyde automatic reuse and in freestanding for-profit units using glutaraldehyde, which accounted for $<5 \%$ of all units. All other interactions of reuse germicide and technique were not different from no reuse.

The varying mortality rates identified in both no-reuse and reuse units using conventional dialysis suggest that other factors, such as dialysis therapy and anemia correction (both known predictors of patient survival), have a greater influence on US mortality than reuse germicides and techniques.

FROM: Collins AJ, Ma JZ, Constantini EG, Everson SE. Dialysis unit and patient characteristics associated with reuse practices and mortality: 1989-1993. J Am Soc Nephrol 1998;9:2108-2117.

\section{Vancomycin-Resistant Enterococci in Australia}

Enterococci with acquired resistance to vancomycin and other glycopeptides have emerged and spread rapidly through Europe and the United States since 1988. Bell and colleagues from the Department of Microbiology and Infectious Diseases, Women's and Children's Hospital, Adelaide, recently reported on the vancomycin-resistant enterococci (VRE) problem in Australia.

The first isolate of VRE in Australia occurred in 1994. Only one case was noted in 1995. Since March 1996, there has been a steady increase in the number of reports of VRE throughout the country. To August 1998, there have been 69 documented strains or clusters of strains detected in patients with documented infection, and approximately three times as many strains have been detected through screening procedures of contacts or in risk groups. Nineteen percent of strains whose source was known were blood isolates, $34 \%$ came from urine, and $47 \%$ came from other specimens. The strains have been found in 26 institutions in 10 widely separated cities or regions of the country (in 6 of 8 states or territories), without any obvious temporal associations in their appearance.

All strains appear to have arisen locally except for one strain imported from the United Kingdom; there was no direct evidence of interhospital transfer of strains. Of the 69 strains, 42 were vanB Enterococcus faecium, 12 were van $\mathrm{E}$ faecium, 9 were van $\mathrm{B}$ Enterococcus faecalis, and 3 were van $\mathrm{A}$ $E$ faecalis. Three were negative for $\operatorname{van} \mathrm{A}, \operatorname{van} \mathrm{B}, \operatorname{van} \mathrm{C} 1$, van $\mathrm{C} 2 / \mathrm{C} 3$, and $\operatorname{van} \mathrm{D}$. Pulsed-field gel electrophoresis (PFGE) profiles on 38 strains have revealed at least 8 types of van $\mathrm{B} E$ faecium, 6 of van $\mathrm{A} E$ faecium, 4 of van $\mathrm{B} E$ faecalis, and 2 of van $\mathrm{A}$ faecalis. Isolates containing van $\mathrm{A}$ always had different profiles from those containing vanB. Clinical clustering was confirmed by PFGE and supported by extended antibiogram. Fourteen of $15 E$ faecalis were ampicillin susceptible, compared to only 2 of $54 E$ faecium. One $E$ faecalis strain was $\beta$-lactamase-positive. The epidemiology of VRE in Australia appears to be different from that of Europe or the United States, since vanB $E$ faecium predominates and strains have appeared in diverse locations independently and are highly polyclonal.

FROM: Bell J, Turnidge J, Coombs G, O'Brien F. Emergence and epidemiology of vancomycin-resistant enterococci in Australia. Commun Dis Intell 1998;22:249-252.

\section{Vancomycin Use in Burn Patients and Risk of Resistance}

Investigators from the US Army Institute of Surgical Research, Fort Sam Houston, conducted a retrospective study to document the risk of the development of vancomycin-resistant bacteria in a population of seriously burned patients during a 10-year period of common vancomycin hydrochloride use. Microbiology, infection, and antibiotic-use records collected during the hospitalization of 2,266 consecutively admitted seriously burned patients were reviewed. Vancomycin was the primary therapeutic agent used for gram-positive infections and also was used as a perioperative prophylactic antibiotic during burn-wound excision. This policy was established because of a high incidence of methicillinresistant Staphylococcus aureus colonization and an anecdotal association of increased $\beta$-lactam resistance in endemic gram-negative pathogens associated with the use of penicillinase-resistant penicillins and cephalosporins.

Examinations of 15,125 gram-positive isolates, including 957 enterococci, for in vitro sensitivity to vancomycin yielded 3 vancomycin-resistant enterococci (VRE) isolates in 3 patients. Vancomycin was used prior to VRE isolation in 1 of these patients. Resistance was found in three other organisms (two Corynebacterium species, 1 Lactobacillus species). Vancomycin was used prior to these isolations in 2 of 3 patients. None of the vancomycin-resistant organ- 
isms was associated with infection, and all 6 patients survived. Vancomycin-resistant enterococci or other vancomycin-resistant gram-positive organisms were not found in 663 patients treated with vancomycin for documented gram-positive infections or in 1,027 patients where perioperative vancomycin was used. It was concluded that the use of vancomycin as the primary therapeutic agent in seriously burned patients was not associated with increased risk of VRE isolation or VRE infection.

FROM: McManus AT, Goodwin CW, Pruitt BA Jr. Observations on the risk of resistance with the extended use of vancomycin. Arch Surg 1998;133:1207-1211.

\section{Survival of Enterococci on Dry Surfaces}

Wendt and coinvestigators from the Institute of Hygiene, Free University, Berlin, compared the abilities of Enterococcus faecium strains (three vancomycin-resistant enterococci [VRE] and five vancomycin-susceptible enterococci [VSE]) and Enterococcus faecalis strains (one VRE and $10 \mathrm{VSE}$ ) to survive under dry conditions. All strains survived for at least 1 week, and two strains survived for 4 months. Survival was not associated with the species ( $E$ faecalis vs $E$ faecium), the source of isolation (patient vs environment), or the susceptibility to vancomycin (VRE vs VSE). Resistance to dry conditions may promote the transmissibility of a strain, but VRE have no advantages over VSE with respect to their ability to survive under dry conditions.

FROM: Wendt C, Wiesenthal B, Dietz E, Rüden H. Survival of vancomycin-resistant and vancomycin-susceptible enterococci on dry surfaces. J Clin Microbiol 1998;36:3734-3736.

\section{Abbreviated Regimens of Zidovudine Reduce Risk of Perinatal HIV Transmission}

The Pediatric AIDS Clinical Trials Group Protocol 076 reported a reduction in the rate of perinatal transmission of HIV from $25.5 \%$ to $8.3 \%$ with a three-part regimen of zidovudine given antepartum, intrapartum, and to the newborn. Wade and colleagues from the New York State Department of Health and the University of Albany, New York, School of Public Health, recently examined the effects of abbreviated zidovudine regimens on perinatal HIV transmission using data from the HIV polymerase chain reaction (PCR) testing service of the New York State Department of Health.

Rates of perinatal HIV transmission varied, depending on when zidovudine prophylaxis was begun. When treatment was begun in the prenatal period, the rate of HIV transmission was $6.1 \%$ (95\% confidence interval, $4.1 \%$ -
$8.9 \%)$; when begun intrapartum, the rate was $10.0 \%(3.3 \%$ $21.8 \%$; when begun within the first 48 hours of life, the rate was $9.3 \%(4.1 \%-17.5 \%)$; and when begun on day 3 of life or later, the rate was $18.4 \%(7.7 \%-34.3 \%)$. In the absence of zidovudine prophylaxis, the rate of HIV transmission was $26.6 \%$ (21.1\%-32.7\%).

These results confirm the efficacy of zidovudine prophylaxis and suggest that there are reductions in the rates of perinatal transmission of HIV even with the use of abbreviated regimens that are begun intrapartum or in the first 48 hours of life. These abbreviated preventive regimens may be particularly suitable for use in parts of the world where the prevalence of HIV infection is high and resources are severely limited.

FROM: Wade NA, Birkhead GS, Warren BL, Charbonneau TT, French PT, Wang L, et al. Abbreviated regimens of zidovudine prophylaxis and perinatal transmission of the human immunodeficiency virus. $N$ Engl J Med 1998;339:1409-1414.

\section{Reducing Vancomycin Use Utilizing a Computer Guideline}

Minimizing vancomycin use represents a key strategy in preventing the spread of vancomycin-resistant enterococci. Shojania and colleagues from Brigham and Women's Hospital, Boston, conducted a study of a computerized guideline to reduce vancomycin use. At the time of physician order entry into the computer, the screen displayed an adaptation of the CDC's guidelines for appropriate vancomycin use. The main outcome measures were the frequency of initiation and renewal of vancomycin therapy and the duration of therapy.

The study included 396 physicians and 1,798 patients in a tertiary-care teaching hospital. Compared with the control group, intervention physicians wrote $32 \%$ fewer orders (11.3 vs 16.7 orders/physician; $P=.04$ ) and had $28 \%$ fewer patients for whom they either initiated or renewed an order for vancomycin ( 7.4 vs 10.3 orders/physician; $P=.02$ ). In addition, the duration of vancomycin therapy attributable to physicians in the intervention group was $36 \%$ lower than the duration of therapy prescribed by control physicians (26.5 vs 41.2 days; $P=05$ ). Pharmacy data confirmed a decrease in the overall hospital use of intravenous vancomycin during the study period. The authors concluded that implementation of a computerized guideline using physician order entry decreased vancomycin use. Computerized guidelines represent a promising tool for changing prescribing practices.

FROM: Shojania KG, Yokoe D, Platt R, Fiskio J, Ma'luf $\mathrm{N}$, Bates DW. Reducing vancomycin use utilizing a computer guideline: results of a randomized controlled trial. J Am Med Inform Assoc 1998;5(6):554-562. 